\title{
The Hogl MAPK prevents cross talk between the HOG and pheromone response MAPK pathways in Saccharomyces cerevísiae
}

\author{
Sean M. O'Rourke and Ira Herskowitz ${ }^{1}$ \\ Department of Biochemistry and Biophysics, University of California, San Francisco, San Francisco, \\ California 94143-0448 USA
}

The MAPKKK Stellp functions in three Saccharomyces cerevisiae MAPK cascades [the high osmolarity glycerol (HOG), pheromone response, and pseudohyphal/invasive growth pathwaysl, but its activation in response to high osmolarity stimulates only the HOG pathway. To determine what restricts cross-activation of MAPK cascades (cross talk), we have studied mutants in which the pheromone response pathway is activated by high osmolarity (1 M sorbitol). We found that mutations in the H OG 1 gene, encoding the p38-type MAPK of the HOG pathway, and in the PBS2 gene, encoding the activating kinase for Hoglp, allowed osmolarity-induced activation of the pheromone response pathway. This cross talk required the osmosensor Sholp, as well as Ste20p, Ste50p, the pheromone response MAPK cascade (Ste11p, Ste7p, and Fus3p or Kss1p), and Stel2p but not Ste4p or the MAPK scaffold protein, Ste5p. The cross talk in hogl mutants induced multiple responses of the pheromone response pathway: induction of a FU S1::lacZ reporter, morphological changes, and mating in ste4 and ste5 mutants. We suggest that Hoglp may prevent osmolarity-induced cross talk by inhibiting Sholp, perhaps as part of a feedback control on the HOG pathway. We have also shown that Ste2Op and Ste50p function in the Sholp branch of the HOG pathway and that a second osmosensor in addition to Sholp may activate Stellp. Finally, we have found that pseudohyphal growth exhibited by wildtype (HOG 1) strains depends on SH 01 , suggesting that Sholp may be a receptor that feeds into the pseudohyphal growth pathway.

[Key Words: Hogl; MAP kinase; yeast pheromone response; yeast high osmolarity response; HOG pathway; signal transduction]

Received May 28, 1998; revised version accepted July 13, 1998.

Multiple mitogen-activated protein kinase (MAPK) cascades coexist in eukaryotic cells and mediate appropriate cellular responses to distinct environmental inputs (Robinson and Cobb 1997; Banuett 1998). The MAPK cascade consists of a MAPK, a MAPK kinase (MAPKK), and a MAPKK kinase (MAPKKK). In Saccharomyces cerevisiae, four complete MAPK cascade modules have been identified (Herskowitz 1995; Levin and Errede 1995). The pheromone response pathway is activated by peptide pheromones and prepares cells for mating (Leberer et al. 1997). The pseudohyphal development/invasive growth pathway responds to environmental conditions to allow formation of pseudohyphal cells in a/ $\alpha$ diploids and invasive growth in haploids (Liu et al. 1993; Roberts and Fink 1994). The PKC-regulated M APK pathway responds to heat stress and hypotonic shock (Davenport et al. 1995; Kamada et al. 1995), and the high osmolarity glyc-

${ }^{1}$ Corresponding author.

E-MAIL ira@cgl.ucsf.edu; FAX (415) 476-0943. erol (HOG) pathway responds to hypertonic stress (Brewster et al. 1993).

The MAPKKK Ste1lp plays an essential role in three of the yeast MAPK pathways: the pheromone response pathway, the pseudohyphal/invasive growth pathway, and the HOG pathway (Fig. 1). In response to pheromones, a heterotrimeric $G$ protein encoded by GPA1 $(\mathrm{G} \alpha)$, STE4 $(\mathrm{G} \beta)$, and STE18 $(\mathrm{G} \gamma)$ is activated, liberating free $G \beta \gamma$ to stimulate downstream components (Dietzel and Kurjan 1987; Miyajima et al. 1987). Free $G \beta \gamma$ binds to and is thought to activate Ste20p, a PAK-related protein kinase (Leeuw et al . 1998), and Ste5p, a scaffold protein that binds each component of the MAPK cascade (Whiteway et al. 1995; Inouye et al. 1997; Feng et al. 1998). The MAPKKK Stellp is activated by a mechanism, probably involving Ste20p-dependent phosphorylation (Wu et al. 1995), which allows Stellp to phosphorylate Ste7p, the MAPKK (N eiman and Herskowitz 1994). Ste7p then phosphorylates and activates the MAPKs Fus3p or Ksslp (Gartner et al. 1992; Errede et al. 


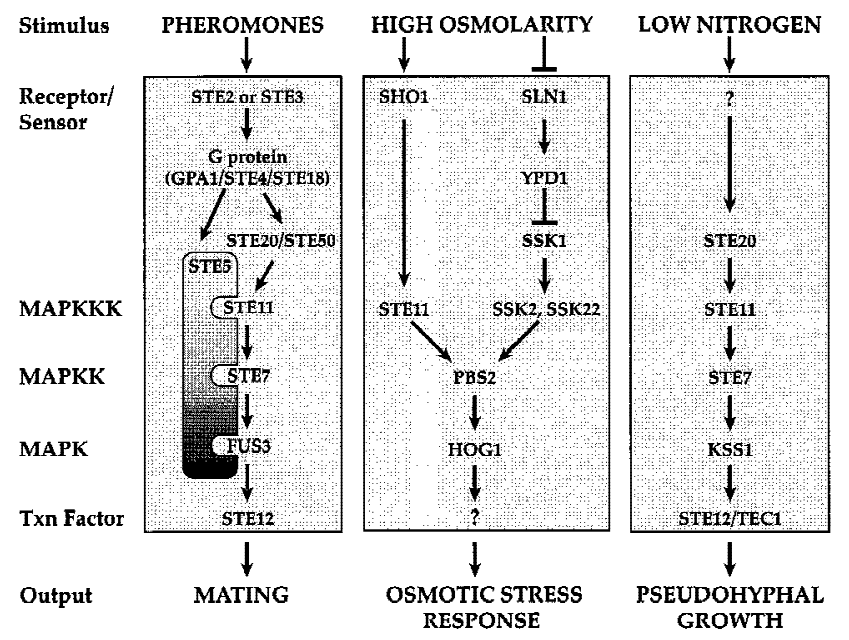

Figure 1. The pheromone response, high osmolarity glycerol (HOG), and pseudohyphal growth pathways.

1993), which activate the transcription factor Ste12p (Cook et al . 1996; Tedford et al. 1997), leading to expression of genes required for mating.

$\mathbf{a} / \alpha$ cells of the $\Sigma 1278 b$ strain background are capable of forming pseudohyphae in response to low nitrogen conditions (Gimeno et al. 1992). Haploid cells of this background exhibit agar invasion on rich medium (Roberts and Fink 1994). Components of the pheromone response pathway are required for pseudohyphal development and invasive growth (Liu et al. 1993; Roberts and Fink 1994): Ste20p, Stellp, Ste7p, and Ste12p are required for both pathways. Ksslp appears to be the MAPK for the pseudohyphal development and invasive growth pathways (Cook et al. 1997; Madhani et al. 1997). The upstream acti vators of these pathways remain to be identified: The pheromone receptors and associated heterotrimeric $\mathrm{G}$ protein are not required for pseudohyphal or invasive growth (Liu et al. 1993; Roberts and Fink 1994). Although the pseudohyphal and invasive growth pathways use a M APK cascade containing Ste11p, Ste7p, and Ksslp, they do not require Ste5p (G. Fink, pers. comm.).

During elevated osmotic conditions, yeast cells accumulate high concentrations of glycerol to counteract the osmotic differential between the inside of the cell and the external environment (for review, see Blomberg and Adler 1992). High osmolarity causes activation of the M APK, Hoglp (Brewster et al. 1993), which triggers increased synthesis of glycerol by activating transcription of genes required for glycerol synthesis such as GPD1 (Larsson et al. 1993; Albertyn et al. 1994). The HOG pathway has two known input branches. One involves a phosphorelay system related to the histidyl-aspartyl phosphorelay systems of bacteria and includes the integral membrane protein SIn1p and the response regulator Ssk 1p (M aeda et al. 1994). Ssk 1p activates two redundant MAPKKKs, Ssk2p and Ssk22p, which subsequently activate Pbs2p, the MAPKK for Hoglp (Maeda et al. 1995; Posas and Saito 1998). The other input is through the osmosensor Sholp, which is a putative membrane-span- ning protein with a carboxy-terminal $\mathrm{SH} 3$ domain (Maeda et al. 1995). This domain binds to a prolinerich re gion in Pbs $2 p$. Activation of Pbs $2 p$ by Sholp requires Ste11p, which phosphorylates Pbs2p. Pbs2p has been termed a scaffold protein because it binds multiple components of the HOG MAPK pathway: Sholp, Stellp, and Hoglp (Posas and Saito 1997). Because there are two inputs for activating Hoglp, mutants defective in either the Sholp branch or the SIn1p branch are not osmosensitive. In contrast, mutants defective in both branches, for example, ssk2 ssk22 sho1 or ssk2 ssk22 ste11 strains are osmosensitive (Maeda et al. 1995; Posas and Saito 1997).

Although Stellp functions in both the pheromone re sponse and HOG pathways, activation of Stellp by high osmolarity does not elicit a mating response (Posas and Saito 1997). Similarly, activation of Stellp by $\alpha$-factor does not activate the HOG pathway (Posas and Saito 1997). The mechanism by which signal specificity is maintained during high osmolarity stress or during exposure to pheromone remains to be determined. The presence of Stellp in two different complexes (with Ste5, Ste7p, and Fus3p or Kss1p or with Sholp, Pbs2p, and Hoglp) has been proposed to be responsible for preventing inappropriate activation of heterologous pathways (Posas and Saito 1997). Here we describe conditions under which the pheromone response pathway is efficiently activated by high osmolarity stress. These studies lead us to propose that Hoglp itself is responsible for limiting cross talk during high osmolarity stress, perhaps by inhibiting the Sholp branch of the HOG pathway. We define the components of the pheromone response and HOG pathways required for cross talk. Our findings al so suggest the existence of a third branch for activating the HOG pathway and raise the possibility that Sholp is an upstream component of the pseudohyphal development pathway.

\section{Results}

O smotic stress induces expression of FUS1::lacZ in Hoglp-deficient strains

In the course of other studies of hogl mutants, which revealed functional relationships between the pheromone response pathway and the HOG pathway (S. O'Rourke, unpubl.), we tested whether $1 \mathrm{~m}$ sorbitol can activate the pheromone response pathway. In our initial experiments, we assayed activation of the pheromone response pathway by induction of the FU S1::lacZ reporter gene, which is activated by Ste12p. Wild-type cells exposed to $1 \mathrm{~m}$ sorbitol did not exhibit any expression of the reporter even after $5 \mathrm{hr}$ of incubation (Fig. 2). In contrast, an isogenic hogl deletion mutant produced a clearly measurable level of $\beta$-galactosi dase by $2 \mathrm{hr}, \sim 20 \%$ the level of these strains exposed to $\alpha$-factor, which increased steadily over $5 \mathrm{hr}$ to reach a level comparable to that observed in the $\alpha$-factor-treated cells (Fig. 2). Wildtype and hogl mutants exhibited similar induction in response to $\alpha$-factor. The presence of both $\alpha$-factor and 


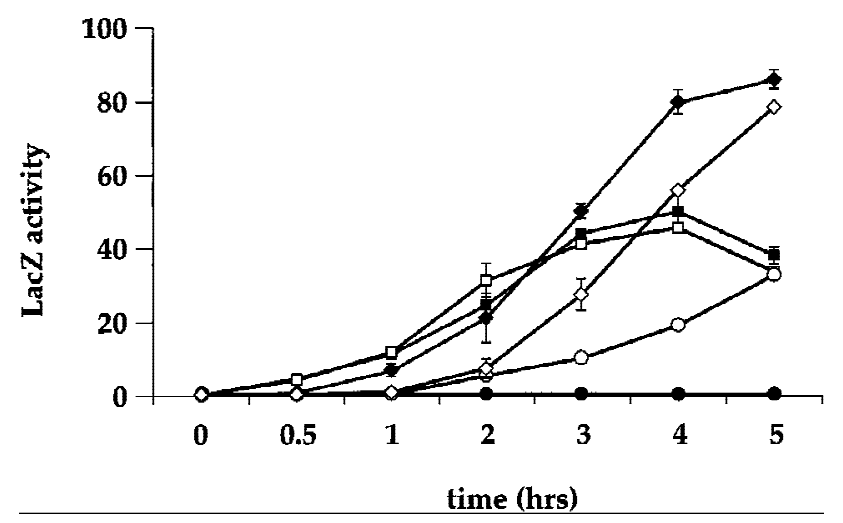

Figure 2. A hogl strain, but not wild type, induces FU S1::lacZ in response to $1 \mathrm{M}$ sorbitol. a wild-type FU S1::lacZ (SO329) (solid symbols) and a hogl FU S1::lacZ (SO330) (open symbols) strains were grown to log phase in YEPD medium, and $t=0$ samples were taken for $\beta$-galactosidase assays. The cells were resuspended in YEPD $+\alpha$-factor medium: ( $\square$ wild type; $\square$ hogl; YEPD $+1 \mathrm{M}$ sorbitol medium: (๑, wild type; $\bigcirc$, hog1), and YEPD +1 M sorbitol $+\alpha$-factor medium: $(\diamond$, wild type; $\diamond$, hog1). At the indicated times, 1-ml samples were harvested for $\beta$-galactosidase assays as described in Materials and Methods. Assays were done in duplicate and the average \pm S.D. of three experiments is shown. LacZ activity is shown in Miller units.

sorbitol led to increased expression of the reporter by 5 hr in both wild-type and hogl strains. Additional experiments showed that $0.5 \mathrm{M} \mathrm{NaCl}$ and $0.4 \mathrm{~m}$ sorbitol also stimulated FU S1::lacZ induction in hogl but not wildtype strains (data not shown). These observations demonstrate that high osmolarity induces the pheromone response pathway in strains lacking Hoglp.

$\mathrm{N}$ ext, we analyzed a series of strains defective in the HOG pathway to determine whether they also exhibit osmolarity-induced activation of FU S1::lacZ (cross talk) (Fig. 3A). M utants gave three different responses: (1) The strain lacking Pbs2p behaved like the hogl mutant, exhibiting high-level induction; (2) mutants defective in either of the known input branches to the HOG pathway, in SHO 1 (affecting the Sholp branch) or in SSK1 or in SSK2 and SSK22 (affecting the SIn1p branch), exhibited negligible cross talk; and (3) mutants defective in both of these branches, because of mutation in both SHO 1 and SSK 1 or in SHO1, SSK2, and SSK 22, exhibited low level s of induction ( $9 \%-15 \%$ of the level observed in hogl and pbs2 mutants). All of these strains exhibited similar induction of FU S1::IacZ in response to $\alpha$-factor. These results indicate that lack of Hoglp activity is required for osmolarity-induced cross talk: Both hogl and pbs2 mutants are completely lacking in Hoglp activity. Because hogl and pbs2 mutants are unable to increase intracellular glycerol in response to osmotic stress, we entertained the possibility that reduction of glycerol due to mutation of GPD1 would also al low cross talk. However, we found the strain deleted for GPD1 did not exhibit cross talk (Fig. 3A).

To determine whether mutants defective in genes other than HOG1 and PBS2 would exhibit osmolarity- induced cross talk, we carried out a screen for mutants that express FU S1::IacZ in the presence of $1 \mathrm{~m}$ sorbitol (see $M$ aterials and M ethods). Of 19 mutants that exhibited $\beta$-gal actosidase activity, 3 were constitutive and were not studied further. Of the 16 remaining mutants, all of which were osmosensitive, 10 were defective in HOG1 and 6 in PBS2 (see Materials and M ethods). The mutant hunt therefore did not identify any genes other than HOG1 and PBS2 that are necessary to restrict cross talk.

Hoglp requires catalytic activity and phosphoacceptor sites to prevent osmolarity-induced cross talk

The studies above showed that strains del eted for HO G1 exhibit osmolarity-induced cross talk. Next, we determined whether mutations that affect specific aspects of Hoglp function also exhibit cross talk. The hogl-K52R mutation is predicted to abolish the catalytic activity of Hoglp; the hogl-T174A and hogl-Y176F mutations block the sites at which Pbs2p phosphorylates Hoglp (Schüller et al. 1994). hogl deletion strai ns carrying plasmids with different HOGI alleles were assayed for FU S1::IacZ expression in the presence of $1 \mathrm{~m}$ sorbitol (Fig. 3B). The strain carrying the wild-type HOG1 plasmid exhibited $\sim 3 \%$ of the full level of cross talk (probably because of plasmid loss). In contrast, the strain producing catalytically inactive Hoglp (hogl-K52R) produced $34 \%$ of the full level of cross talk. The hoglY176F and hog1-T174A mutants exhibited even higher levels (58\% and $102 \%$, respectively). The two mutants that exhibited lower levels of cross talk (hogl-K52R and hogl-Y176F) appear to retain some Hoglp activity, as they can support growth in the presence of intermediate osmolarity (Schüller et al. 1994). These observations indicate that catalytic activity of Hoglp is required to inhibit cross talk.

Osmolarity-induced cross talk requires components of the pheromone response pathway downstream of Ste4p, but not Ste5p

Activation of the FU S1::IacZ reporter by high osmolarity in Hoglp-deficient strains could in principle occur by activation of the pheromone response pathway at any level, from the receptor, Ste2p, to the transcription factor, Ste12p. To determine at what level the signal from high osmol arity feeds into the pheromone response pathway, we analyzed cross tal $k$ in hogl mutants defective in different components of the pheromone response pathway (Fig. 3C). These hogl strains exhibited two types of behavior: Those defective al so in STE4 or STE5 behaved like a hogl single mutant and exhibited an essentially full level of cross talk ( $74 \%$ and $81 \%$, respectively). In contrast, hogl strains also defective in STE20, STE50, STE11, STE7, STE12, or in both FUS3 and KSS1 exhibited no cross tal $k(<1 \%)$. hogl mutants defective in either FU S3 or KSS1 exhibited osmolarity-induced cross talk like the hogl single mutant (data not shown), indicating that Fus3p and Kss1p are functionally redundant for this 
A

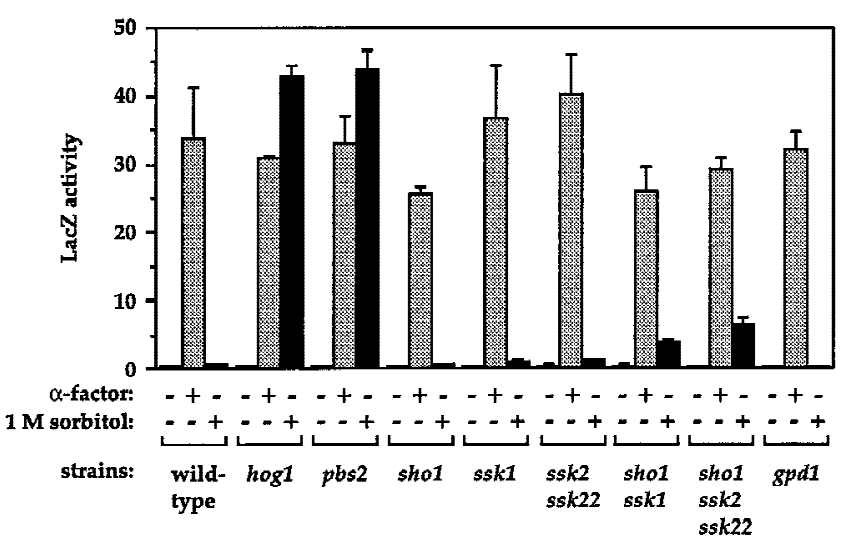

C

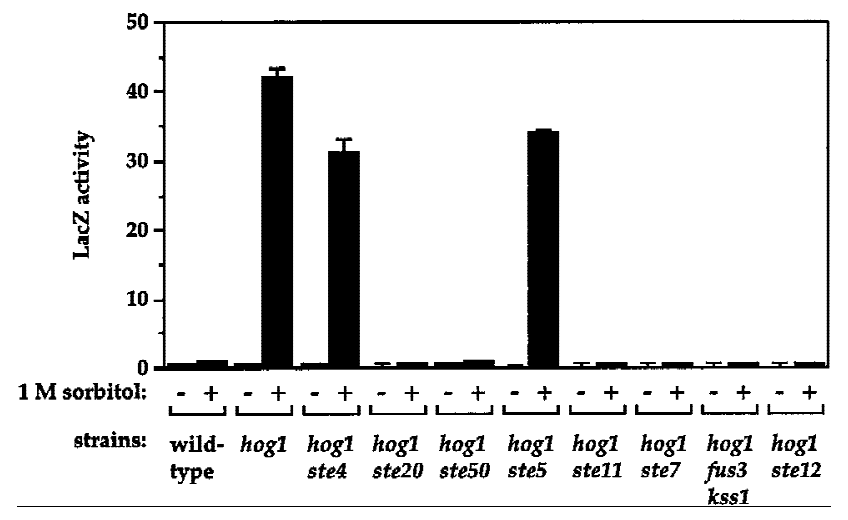

B

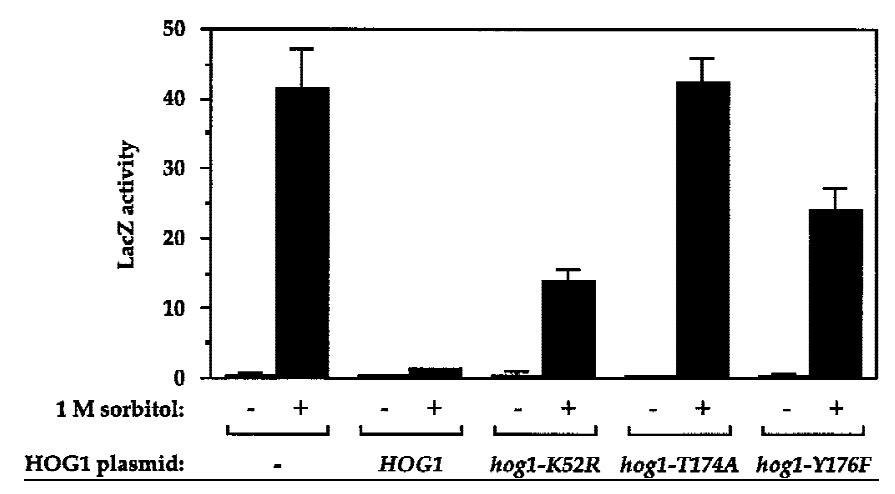

$\mathbf{D}$

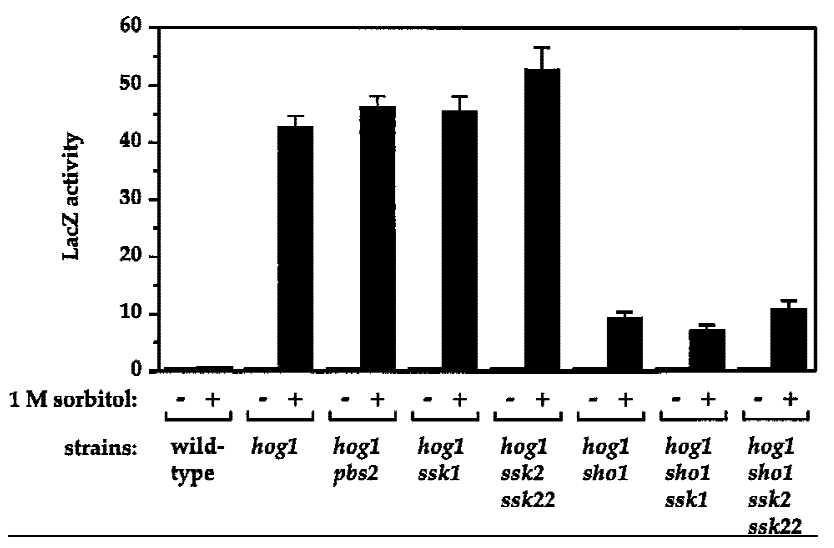

Figure 3. Genetic control of osmolarity-induced cross talk using FU S1::lacZ as a reporter. Cultures of log phase yeast strains were shifted to the indicated media for $5 \mathrm{hr}(6 \mathrm{hr}$ for B) and harvested. $\beta$-Galactosidase assays were done in triplicate, and the average \pm S.D. of three experiments is shown. LacZ activity is plotted in Miller units. (A) Dependence of osmolarity-induced cross talk on inactivation of the HOG pathway. Strains SO 329 (wild type), SO330 (hog1), SO382 (pbs2), SO351 (sho1), SO352 (ssk1), SO354 (ssk2 ssk22), SO 353 (shol ssk1), SO 355 (shol ssk2 ssk22), and SO 385 (gpd1) were tested. (B) Hoglp kinase activity and phosphoacceptor sites are required to prevent cross-activation of FU S1::IacZ in response to $1 \mathrm{M}$ sorbitol. Strain SO330 (hogl FU S1::IacZ) was transformed with an empty vector (pRS316) or low-copy HOG1-containing plasmids: HOG1 (pJB15), hog1-K52R (pJBM 3), hog1-T174A (pJBM 4), or hog1-Y176F (pJBM 2). Two independent transformants were assayed for each plasmid. (C) Dependence on the pheromone response pathway for osmolarity-induced cross talk in hogl mutants. Strains SO329 (wild type), SO330 (hogl), SO331 (hogl ste4), SO391 (hogl ste20), SO 373 (hogl ste50), SO 332 (hogl ste5), SO333 (hogl stel1), SO334 (hogl ste7), SO 335 (hogl fus3 kss1), and SO143 (hogl ste12) were analyzed. (D) Dependence on the Sholp osmosensor for osmolarity-induced cross talk in hogl mutants. The strains tested were SO 329 (wild type), SO330 (hog1), SO 383 (hog1 pbs2), SO384 (hogl ssk1), SO 358 (hogl ssk2 ssk22), SO 356 (hog1 sho1), SO 357 (hog1 shol ssk1), SO359 (hogl shol ssk2 ssk22).

pathway (see Cook et al. 1997; Madhani et al. 1997). These observations show that the pheromone response MAPK cascade and downstream transcription factor Ste12p are necessary for osmolarity-induced cross talk, as are Ste20p and Ste50p, which play an imperfectly defined role in activating Stellp (Ramer and Davis 1993; Wu et al . 1995; Xu et al . 1996). In contrast, Ste4p, the G $\beta$ component of the heterotrimeric $G$ protein, and Ste5p, the scaffold for the M APK module, are not required. $\mathbf{a} / \alpha$ hogl/hogl di ploids, which do not express STE4 or STE5 (see Bardwell et al. 1994), al so exhibit cross talk (data not shown). These observations indicate that the signal for activating transcription of FU S1::lacZ in hogl mutants enters the pheromone response pathway after Ste4p and Ste5p and prior to Ste20p and Ste50p.

Only one of the known osmosensors, Sholp, is required for osmolarity-induced cross talk

Because activation of the pheromone response pathway in hogl and pbs2 mutants occurs in response to osmotic stress rather than being constitutive, we anticipated that components of the HOG pathway involved in sensing high osmolarity stress would be required for induction. To identify these components, we tested strains defective in both HOGI and in additional genes of the HOG 
pathway. Strains defective in both HOG1 and PBS2, as expected, behaved the same as singly defective strains (Fig. 3D). Inactivating the SIn1p branch of the pathway, as in hogl ssk 1 and hogl ssk 2 ssk 22 strains, similarly did not affect the level of cross talk. In contrast, hogl strains defective in $\mathrm{SHO} 1$ exhibited a $78 \%$ reduction in cross talk. Cross talk exhibited by pbs 2 mutants was similarly reduced by a mutation in SHO 1 (data not shown). Inactivation of the SInlp branch in the hogl shol strain by deleting SSK 1 or SSK 2 and SSK 22 did not reduce cross talk further. These results indicate that the major source of the high osmolarity signal that triggers the pheromone response pathway in hogl mutants is the Sholp branch, which shares components with the pheromone response pathway (see below). The observation that osmolarityinduced cross talk in hogl shol ssk 1 mutants remains at $16 \%$ of the full level, much higher than observed in hogl stell or hogl ste50 mutants (Fig. 3C), leads us to propose the existence of a third osmosensing branch in addition to Sholp and SIn1p (see Discussion).

High osmolarity induces shmoo formation and mating in hogl mutants

The pheromone response pathway governs not only induction of FU S1::IacZ but also mediates cellular morphology changes and mating. To determine whether osmolarity-induced activation of the pheromone response pathway exhibited by hogl mutants extends to these processes, we first examined the ability of high osmolarity to induce morphological alterations (formation of pear-shaped cells, shmoos) characteristic of pheromonetreated cells. Shmoos formed by wild-type yeast in response to $\alpha$-factor are shown in Figure 4 (middle row), in contrast to the budded morphology of cells grown in the absence of pheromone (Fig. 4, top row). Exposure of wildtype yeast cells to $1 \mathrm{~m}$ sorbitol had no effect on their morphology (Fig. 4, bottom row). In contrast, 1 м sorbitol induced hogl and pbs2 mutants to form shmoo-like cells, a response that required functional $\mathrm{SHO} 1$ and STE12 genes (Fig. 4, bottom row). In other studies, we have observed that STE20, STE11, STE7, and FU S3 or KSS1 are required for this morphological response in hog1 strains, whereas STE4, STE5, PBS2, SSK1, SSK2, and SSK22 are not (S. O'Rourke, unpubl.). A strain defective in SSK 1 and SHO 1 did not exhibit a morphological change in response to $1 \mathrm{~m}$ sorbitol (Fig. 4, bottom row), just as this strain did not exhibit a high level of FU S1::IacZ induction when stimulated with $1 \mathrm{~m}$ sorbitol.

Having found that high osmolarity can induce expression of FU S1::IacZ and a shmoo-like morphol ogy in hogl mutants, we tested whether high osmolarity could al so restore mating to hogl strains also defective in various genes required for mating. Wild-type and hogl strains mated efficiently in both the presence and absence of $1 \mathrm{M}$ sorbitol (Table 1). As expected, HOG1 strains defective in STE4, STE5, STE11, STE7, STE12, or both FU S3 and KSS1 were defective in mating in the presence or absence of $1 \mathrm{~m}$ sorbitol (Table 1; data not shown); hogl strains defective in different STE genes exhibited three different responses: (1) hogl strains defective in STE11, STE7, STE12, or FUS3 and KSS1 were defective in mating both in the presence and absence of $1 \mathrm{~m}$ sorbitol (T able 1; data not shown); (2) the substantial residual mating exhibited by hogl ste20 strains ( $10 \%$ that of hogl STE20 strains) was reduced $>70$-fold by $1 \mathrm{~m}$ sorbitol (1 M sorbitol al so reduced mating of the HOG 1 ste20 strain 4-fold); and (3) mating by hogl ste4 was increased 670 -fold in the presence of $1 \mathrm{~m}$ sorbitol, from $2.7 \times 10^{-7}$ to $1.8 \times 10^{-4}$. An even more striking improvement in mating was displayed by the hogl ste5 strain, which exhibited a $16,000-$ fold increase in mating in the presence of $1 \mathrm{~m}$ sorbitol, from $1.6 \times 10^{-7}$ to $2.6 \times 10^{-3}$. The improved mating due to $1 \mathrm{~m}$ sorbitol resulted from osmolarity-induced cross talk, as it was dependent on SHO 1: The hogl shol ste4 strain mated equal ly poorly in the absence or presence of $1 \mathrm{~m}$ sorbitol, and the hogl shol ste5 strain exhibited only a threefold improvement in mating in the presence of 1 M sorbitol. Interestingly, 1 m sorbitol slightly improved mating by a HOG1 ste4 strain (a 4.5-fold incease), suggesting that a low level of osmolarity-induced cross talk might al so occur in HOGl cells. Thus, the mating assays parallel our results obtained with the FUS1::IacZ and morphological assays of cross talk: hogl strains defective in STE4 or STE5 exhibit osmolarity-induced cross talk, but hogl strains defective in SHO1, STE20, or downstream pheromone response pathway genes do not exhibit cross talk.

\section{Ste50p and Ste20p are components} of the Sholp-dependent branch of the HOG pathway Because STE50 and STE20 are required for osmolarity-
Figure4. High osmolarity induces a shmoolike morphology in hogl and pbs2 mutants. (Top row) Cells incubated in YEPD liquid medium; (middle row) cells treated with $\alpha$ factor in YEPD for $2 \mathrm{hr}$; (bottom row) cells treated with $1 \mathrm{~m}$ sorbitol in YEPD for $6 \mathrm{hr}$. Strains SO329 (wild type), SO330 (hogl), SO382 (pbs2), SO353 (ssk1 sho1), SO143 (hogl stel2), and SO356 (hogl shol) were grown to log phase and shifted into fresh liquid media as indicated.

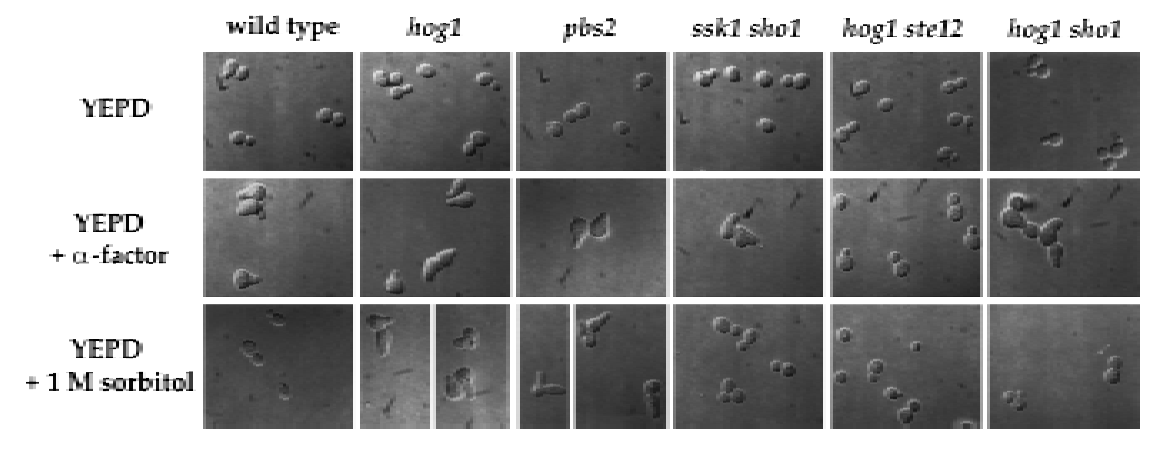


Table 1. A hogl mutation suppresses the mating defects of ste 4 and ste 5 strains in the presence of $1 \mathrm{~m}$ sorbitol

\begin{tabular}{|c|c|c|c|}
\hline a strains ${ }^{a}$ & YEPD mating $(\%)^{\mathrm{b}}$ & $\begin{array}{c}\text { YEPD }+1 \mathrm{M} \text { sorbitol } \\
\text { mating }(\%)^{\mathrm{b}}\end{array}$ & $\begin{array}{c}\text { Effect of } 1 \mathrm{M} \\
\text { sorbitol }^{\mathrm{C}}\end{array}$ \\
\hline $\begin{array}{l}\text { Wild type } \\
\text { ste4 } \\
\text { ste5 } \\
\text { ste20 } \\
\text { stel1 }\end{array}$ & $\begin{aligned} & 58 \pm 5 \\
& 1.4 \pm 0.4 \times 10^{-5} \\
& 4.7 \pm 2 \times 10^{-6} \\
& 2.9 \pm 0.6 \\
&<3.3 \pm 2 \times 10^{-6}\end{aligned}$ & $\begin{aligned} 74 & \pm 12 \\
6.8 & \pm 2 \times 10^{-5} \\
6.6 & \pm 2 \times 10^{-6} \\
0.79 & \pm 0.3 \\
5.3 & \pm 2 \times 10^{-6}\end{aligned}$ & $\begin{array}{l}1.3 \\
4.9 \\
1.4 \\
0.27 \\
-\end{array}$ \\
\hline $\begin{array}{l}\text { hogl } \\
\text { hogl ste4 } \\
\text { hogl ste5 } \\
\text { hogl ste20 } \\
\text { hogl stell }\end{array}$ & $\begin{array}{l}68 \pm 7 \\
2.7 \pm 1 \times 10^{-5} \\
1.6 \pm 0.6 \times 10^{-5} \\
6.6 \pm 2 \\
<3.8 \pm 1 \times 10^{-6}\end{array}$ & $\begin{aligned} 63 & \pm 9 \\
1.8 & \pm 0.3 \times 10^{-2} \\
0.26 & \pm 0.08 \\
9.4 & \pm 3 \times 10^{-2} \\
1.2 & \pm 0.7 \times 10^{-5}\end{aligned}$ & $\begin{array}{c}0.93 \\
670 \\
16,000 \\
0.014 \\
-\end{array}$ \\
\hline $\begin{array}{l}\text { hogl shol } \\
\text { hogl shol ste } 4 \\
\text { hogl shol ste5 }\end{array}$ & $\begin{array}{l}72 \pm 8 \\
3.5 \pm 2 \times 10^{-5} \\
2.0 \pm 2 \times 10^{-5}\end{array}$ & $\begin{array}{l}61 \pm 9 \\
3.1 \pm 0.8 \times 10^{-5} \\
6.1 \pm 4 \times 10^{-5}\end{array}$ & $\begin{array}{l}0.85 \\
0.89 \\
3.1\end{array}$ \\
\hline
\end{tabular}

a Strains tested were SO329 (wild type), SO341 (ste4), IH 2731 (ste5), IH2735 (ste20), SO336 (stel1), SO330 (hogl), SO331 (hog1 ste4), SO332 (hogl ste5), SO391 (hogl ste20), SO 333 (hogl ste11), SO356 (hogl sho1), SO344 (hogl shol ste4), and SO398 (hogl shol ste5). bMating efficiency is the number of diploids formed divided by the number of total cells at the end of the experiment. Strains were mated to wild-type $\alpha$ strain IH 1793.

'The effect of $1 \mathrm{~m}$ sorbitol was calculated by dividing the mating efficiency on YEPD $+1 \mathrm{~m}$ sorbitol by the mating efficiency on YEPD.

induced cross talk (Fig. 3C) and function upstream of Stellp, we reasoned that these genes may normally function in the HOG pathway. The role of STE11 in the HOG pathway is revealed in mutants that are defective al so in the SI n1p branch: stell ssk 2 ssk 22 and stell ssk 1 mutants are osmosensitive, as is a shol ssk1 strain (Posas and Saito 1997; see below). As shown in Figure 5A, wild-type, ssk1, sho1, ste50, and ste20 strains grew equally well on YEPD and YEPD $+\mathrm{NaCl}$ plates. In contrast, the ste50 ssk 1 double mutant was osmosensitive on YEPD $+1 \mathrm{~m} \mathrm{NaCl}$ plates, as was the shol ssk1 double mutant. The ste50 shol double mutant was as osmoresistant as the ste50 and shol single mutants (data not shown), suggesting that Ste50p functions specifically in the Sholp-dependent branch of the HOG pathway. As described previously, a ste20 mutation did not increase osmosensitivity when present in a strain defective for the SIn1p branch (Posas and Saito 1997). We verified this result in our strain background for growth on YEPD +1 $\mathrm{M} \mathrm{NaCl}$ medium. However, we observed that the ssk1 ste20 strain was osmosensitive on YEPD $+1.2 \mathrm{M} \mathrm{N} \mathrm{aCl}$ medium (Fig. 5A). ste50 and ste20 mutations al so caused osmosensitivity in strains lacking SSK 2 and SSK22 (data not shown).

Because osmosensitivity can arise from a variety of physiological defects and not necessarily because of inability to activate Hoglp, we tested the role of Ste20p and Ste50p in the HOG pathway by assessing phosphorylation of Hoglp directly. As described previously, incubation of yeast cells in high osmolarity medium results in tyrosine phosphorylation of Hoglp (Brewster et al. 1993). We utilized an anti-phospho-specific p38 antibody that recognizes the phosphorylated form of p38 to detect Hoglp activation. In Figure 5B, we show that the antibody recognized phosphorylated Hoglp in extracts from wild-type yeast treated with $0.7 \mathrm{~m} \mathrm{NaCl}$ for $5 \mathrm{~min}$, but not in untreated cells or in extracts prepared from $\mathrm{N} \mathrm{aCl}$ - treated hog1 or pbs2 mutants. As shown previously for ssk2 ssk22 strains (M aeda et al. 1995) and in Figure 5B for ssk1 strains, Hoglp is phosphorylated efficiently in mutants defective in the $\operatorname{SIn} 1 \mathrm{p}$ branch, presumably by the Sholp pathway. However, when SHO 1, STE20, STE50, or STE11 is deleted in an ssk1 mutant, Hoglp was not phosphorylated during exposure to $0.7 \mathrm{M} \mathrm{NaCl}$ (Fig. 5B). The inability to phosphorylate Hoglp and the osmosensitivity data indicate that Sholp, Ste20p, Ste50p, and Stellp all function to transduce the osmotic stress signal to Hoglp during high extracellular osmolarity. In contrast, we found no role for Ste7p in the HOG pathway: A ste7 ssk 1 double mutant phosphorylated Hoglp in response to $0.7 \mathrm{M} \mathrm{N} \mathrm{aCl}$ and was osmoresistant on medium containing $1.2 \mathrm{M} \mathrm{N} \mathrm{aCl}$ (data not shown).

SHO1 is required for pseudohyphal growth in wild-type and Hoglp-deficient strains

$a / \alpha$ strains of the $\Sigma 1278 b$ background exhibit pseudohyphal growth when starved for nitrogen (on SLAHD medium; Gimeno et al. 1992). Pseudohyphal development requires STE20, STE11, STE7, KSS1, and STE12 but not STE4, STE5, or STE18 (Liu et al. 1993; Cook et al. 1997; Madhani et al. 1997; G. Fink, pers. comm.). a/ $\alpha$ hogl/ hogl strains were reported recently to exhibit a hyperpseudohyphal phenotype on SLAHD medium, which is at low osmolarity (M adhani et al. 1997; Fig. 6A, B). We reasoned that Sholp-dependent cross talk may be responsible for this hyperpseudohyphal growth. The $\mathbf{a} / \alpha$ hogl/hogl shol/shol strain exhibited greatly reduced pseudohyphal development (Fig. $6 \mathrm{D}$ ): Only $0.5 \%$ of the colonies had extensive pseudohyphae, compared to $84.5 \%$ of col onies produced by the isogenic $\mathrm{SHO} 1 / \mathrm{SHO} 1$ strain (Table 2). The hyperpseudohyphal growth of hog1/ hogl strains thus requires function of Sholp under conditions of low osmolarity. We observed furthermore that 
A
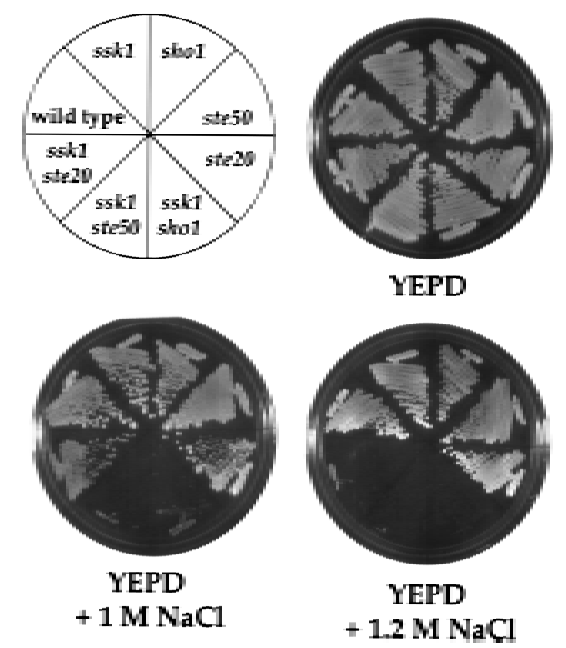

B

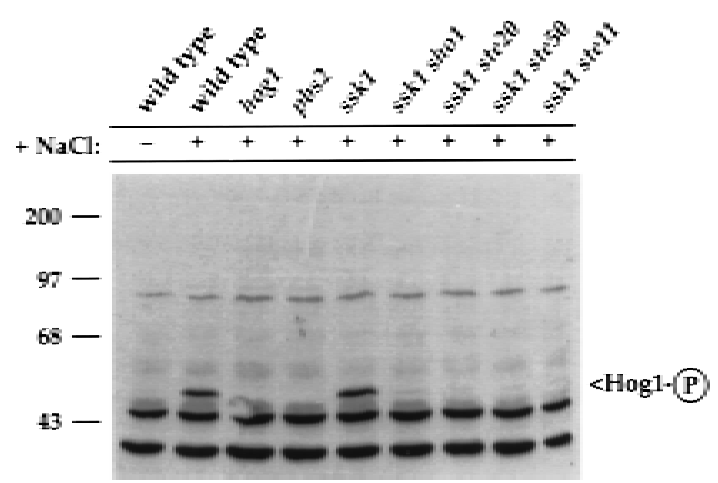

Figure 5. Ste50p and Ste20p function in the Sholp branch of the HOG pathway. (A) Osmosensitivity of ste50 and ste20 mutants. Y east strains were streaked on YEPD, YEPD $+1 \mathrm{~m} \mathrm{NaCl}$, and YEPD $+1.2 \mathrm{M} \mathrm{N} \mathrm{aCl}$ plates, as indicated, and grown for 3 days (YEPD), 6 days (YEPD $+1 \mathrm{~m} \mathrm{NaCl}$ ) or 8 days (YEPD $+1.2 \mathrm{~m}$ $\mathrm{NaCl}$ ), at $30^{\circ} \mathrm{C}$ to assay growth. SO329 (wild type), SO352 (ssk1), SO 351 (sho1), SO 360 (ste50), IH 2735 (ste20), SO 353 (ssk1 sho1), SO361 (ssk1 ste50), and SO 387 (ssk1 ste20) were examined. The osmosensitivities of the ste50 ssk 1 and ste20 ssk 1 strains were complemented by low-copy STE50 and STE20 plasmids, respectively (data not shown), indicating that the phenotypes are attributable to the ste50 and ste20 mutations. (B) Ste50p- and Ste20p-dependent activation of Hoglp in an SSK1deficient strain. Y east cultures were grown in YEPD and treated with $0.7 \mathrm{~m} \mathrm{NaCl}$ for $5 \mathrm{~min}$, as indicated, prior to preparing total protein. The presence of activated Hoglp was visualized by probing a blot with an anti-phospho-specific p38 antibody as described in $M$ aterials and $M$ ethods. M olecular size markers are shown in KD. Strains analyzed were SO 329 (wild type), SO 330 (hog1), SO382 (pbs2), SO352 (ssk1), SO353 (ssk1 sho1), SO387 (ssk1 ste20), SO361 (ssk1 ste50), and SO399 (ssk1 stel1).

HOG 1 strains also require SHO 1 for pseudohyphal development (Fig. $6 \mathrm{C}$ ): Only $2 \%$ of the colonies formed by the HOG1/HOG1 shol/shol strain exhibited robust

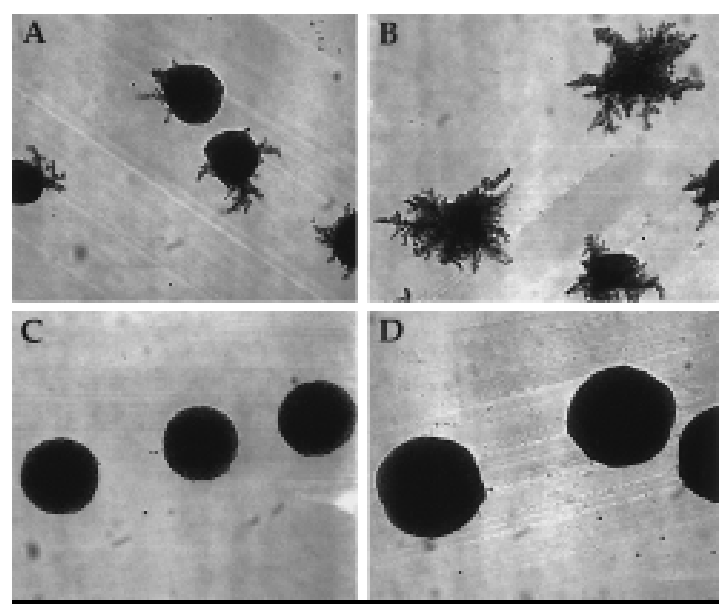

Figure 6. Hyperfilamentous growth of hogl/hogl a/ $\alpha \Sigma 1278 b$ strains is dependent on SHO1. (A) wild type (SO392) +YCplac22, (B) hogl/hogl (SO393) +YCplac22, (C) shol/shol (SO394), and (D) hogl/hogl shol/shol (SO395) strains in the $\Sigma 1278 \mathrm{~b}$ genetic background were streaked on a SLAHD plate and grown for 5 days.

pseudohyphal development, and 92.5\% lacked pseudohyphae altogether (Table 2 ). In contrast, $43 \%$ of wildtype colonies exhibited pseudohyphae like those in Figure $6 \mathrm{~A}$, and $31.5 \%$ lacked pseudohyphae. These observations demonstrate that $\mathrm{SHO} 1$ is necessary both for the hyperpseudohyphal response of hogl/hogl strains as well as for normal pseudohyphal development in wildtype strains.

\section{Discussion}

The presence of Stellp in three different MAPK pathways (the pheromone response pathway, the pseudohyphal development pathway, and the HOG pathway; see Fig. 7) poses the problem of cross talk acutely: What is the mechanism that restricts Stellp to activate only a subset of potential targets? We have found that Hoglp, the MAPK of the HOG pathway, limits activation of the pheromone response pathway by high osmolarity (cross talk). In particular, hogl mutants exhibit inappropriate signaling (diagrammed as a dashed line in Fig. 7), which is manifested in several different ways: activation of

Table 2. SHOl is required for pseudohyphal growth

\begin{tabular}{lccc}
\hline & \multicolumn{3}{c}{ Filamentation (\% of total colonies) } \\
\cline { 2 - 4 } Strains $^{\text {a }}$ & robust & weak & none \\
\hline Wild type & 43 & 25.5 & 31.5 \\
hog1/hog1 & 84.5 & 15 & 0.5 \\
sho1/sho1 & 2 & 6 & 92.5 \\
hog1/hog1 sho1/sho1 & 0.5 & 15 & 84.5 \\
\hline
\end{tabular}

a/ $\alpha \Sigma 1278 b$ strains assayed were wild type (SO392), hog1/hogl (SO393), shol/shol (SO394), and hogl/hogl shol/shol (SO395). Two hundred colonies were counted for each strain. The SO392 and SO 393 strains harbored plasmid Y C plac22 to complement the trp1 auxotrophy in these strains. Robust, as in Fig. 6, A and B; weak, one filament per colony. 


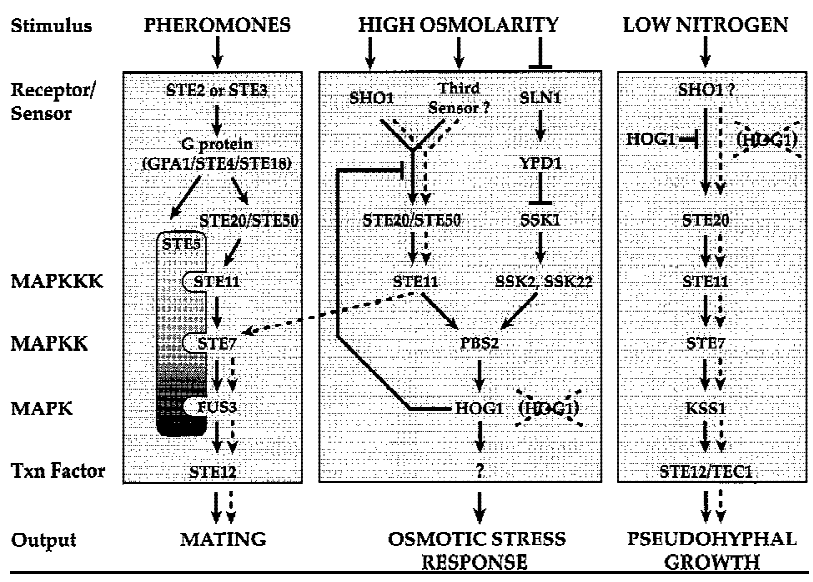

Figure 7. A revised model of the pheromone response, HOG, and pseudohyphal growth pathways. The diagram shows feedback regulation of the Sholp branch of the HOG pathway by Hoglp. Ste50p and Ste20p are shown as components of both the pheromone response and HOG pathways. Sholp is shown as a sensor for both the pseudohyphal growth and HOG pathways. Processes that occur inappropriately in hogl mutants (osmolarity-induced cross talk to the pheromone response pathway and hyperpseudohyphal growth) are shown as broken lines.

FU S1::IacZ, induction of shmoo morphology, suppression of mating defects in ste4 and ste5 mutants, and hyperpseudohyphal growth. Our studies have focused on three aspects of this phenomenon. First of all, we have identified the components of the HOG pathway and the pheromone response pathway that are required for osmolarity-induced cross talk in hogl mutants. Second, we have exploited features of this cross tal $k$ phenomenon to reveal a role for Ste50p and Ste20p in the HOG pathway and to suggest the existence of a third input to the HOG pathway. Finally, our experiments with $\Sigma 1278 \mathrm{~b}$ strains suggest that Sholp provides input not only for the HOG pathway but for the pseudohyphal pathway as well.

O smolarity-induced cross talk uses components of the HOG pathway, including some newly identified components

Osmolarity-induced activation of the pheromone response pathway exhibited by hogl mutants begins with an osmosensing component of the HOG pathway (Sholp) and culminates with the pheromone response MAPK cascade. The importance of Sholp is demonstrated by the observation that mutation of $\mathrm{SHO} 1$ causes a 78\% reduction in the level of cross talk in hogl strains. The SIn 1p branch of the HOG pathway apparently plays no role in osmolarity-induced cross talk: hogl mutants defective in SSK 1 or in SSK2 and SSK22 still exhibit high levels of cross talk.

The finding that Ste50p and Ste20p are required for osmolarity-induced cross talk in hogl mutants led us to test their role in the HOG pathway of wild-type cells. Such a role could be seen in strains lacking the SIn1p branch of the HOG pathway as measured by osmosensitivity and phosphorylation of Hoglp (Fig. 5). In particu- lar, a STE50 mutation caused osmosensitivity in a strain lacking SSK1. Although prior studies (Posas and Saito 1997) had failed to find a requirement of STE20 in osmotolerance, we observed that ste20 ssk 1 strains are much more sensitive than STE20 ssk 1 strains to $1.2 \mathrm{M} \mathrm{N} \mathrm{aCl}$. In addition to being osmosensitive, the ste20 ssk 1 and ste50 ssk 1 strains similarly failed to activate Hoglp during high osmolarity stress, as was found previously for strains defective for both the SIn1p branch (ssk2 ssk22) and SHO 1 or STE11 (Posas and Saito 1997). These observations indicate that Ste20p and Ste50p are involved in activation of Stellp in both the HOG and pheromone response pathways.

\section{The HOG pathway may have a third input branch}

Three different observations lead us to propose the existence of a third input branch to the HOG pathway. First, in a hogl background, eliminating the two known input branches to the pathway, by mutation in SHOl and SSK 1 or in SHO1, SSK2, and SSK22, reduced but did not el iminate osmolarity-induced cross talk. Second, we observed that shol ssk 1 and shol ssk 2 ssk 22 strains exhibited a significant level of cross talk. Because the cross talk observed in these strains is osmolarity-induced, it is clear that an osmosensing function remains active even in the absence of the two known inputs to the HOG pathway. Finally, we observed that stell ssk 1 and ste50 ssk1 strains display a stronger osmosensitive phenotype than shol ssk 1 strains (S. O'Rourke, unpubl.), suggesting that the hypothetical third input to the HOG pathway feeds into Stellp and Ste50p.

O smolarity-induced cross talk uses some elements of the pheromone response pathway, but not Ste5p

As expected, osmolarity-induced cross talk utilizes components of the pheromone response pathway in addition to those shared with the HOG pathway: The MAPK module and the transcription factor Ste12p are also re quired. Given that Sholp is thought to normally activate Stellp for the osmotic stress response, it is not surprising that a more upstream component of the pheromone response pathway, G $\beta$ (Ste4p), is not required for cross talk. It is striking that Ste5p is not required for osmolarity-induced activation of the pheromone response pathway reporter FU S1::IacZ, given that it is required when the pheromone response pathway MAPK cascade, composed of Stellp, Ste7p, and the M APK Fus3p, is activated by pheromone (M cCaffrey et al. 1987). This observation parallels the finding that the M APK module of the pseudohyphal development pathway, containing Stellp and Ste7p al ong with the M APK Kssip, al so does not require Ste5p (G. Fink, pers. comm.). These observations lead us to propose that Sholp may perform some of the same functions as Ste5p, in particular, for linking the activation of Stellp to the external environment and for activation of Ste7p. As discussed further bel ow, we propose that Sholp performs these functions not only during osmolarity-induced cross talk in hogl mutants, but also during pseudohyphal growth in wild-type (HOG1) cells. 
hogl mutants induce multiple STE12-dependent processes

High osmolarity induces all of the same processes in hogl mutants as are induced by mating pheromones in wild-type cells. The classical reporter for transcriptional activation, FU S1::lacZ, is induced more slowly by $1 \mathrm{~m}$ sorbitol than by $\alpha$-factor, but the levels of induction are comparable after a few hours (Fig. 2). Likewise, we observed that $1 \mathrm{~m}$ sorbitol induces hogl and pbs2 mutants to form projections similar to those induced by $\alpha$-factor. Perhaps most strikingly, high osmol arity improved mating by ste4 hogl and ste5 hogl mutants 670- and 16,000fold, respectively (Table 1 ). The greater increase seen in the ste 5 mutant than in the ste 4 mutant may be due to roles of Ste4p during mating in addition to stimulating the MAPK module (Schrick et al. 1997). The ability to activate the pheromone response MAPK module in the absence of Ste4p and Ste5p using sorbitol as a mating pheromone offers some novel opportunities to dissect early steps in the mating pathway.

In contrast to the ability of high osmolarity to improve mating in ste4 hogl and ste5 hogl mutants, $1 \mathrm{~m}$ sorbitol did not suppress the mating defect of stell hogl, ste7 hogl, fus3 kss1 hogl, or stel2 hogl strains. This lack of suppression is consistent with the idea that the cross talk signal enters the pheromone response pathway upstream of Stellp.

We observed that the hyperpseudohyphal growth of hogl/hogl strains (M adhani et al. 1997) is dependent on SHO 1 and thus may represent osmolarity-independent activation of Stellp by Sholp. To our surprise, we observed that $\mathrm{SHO} 1$ is also required for pseudohyphal development in wild-type (HOG1) strains. These results indicate that Sholp provides input to the pseudohyphal development pathway not only under the unnatural conditions of a hoglp-mutant strain but in wild-type strains as well. According to this view, the hyperpseudohyphal growth of hogl/hogl strains would not represent cross talk but, rather, constitutive activation of the pathway by loss of a negative regulator (HOG1). It will be of interest to determine whether Sholp and Hoglp respond not only to osmotic stress but to other stimuli as well. In Schizosaccharomyces pombe, the pathway that senses high osmolarity responds to multiple stimuli, including nutrient limitation (for review, see Banuett 1998).

Hoglp may down-regulate the Sholp branch of the HOG pathway to limit cross talk

It has been proposed that the presence of Stellp in different protein complexes (with Ste7p, Fus3p, and Ste5p and with Pbs2p, Hoglp, and Sholp) restricts cross activation (Posas and Saito 1997). Activated Stellp might phosphorylate only the MAPKK (Ste7p or Pbs2p) present within its complex. Evidence that Ste5p can sequester Ste7p comes from the observation that the ability of certain forms of Ste7p to partially bypass a mutation in the PKC pathway (bck1) is greater in ste5 mutants than in STE5 strains (Yashar et al. 1995). We have shown that high levels of cross talk are observed in the complete absence of Hoglp or with mutant proteins that are defective in its catalytic activity or in its activation. Because the hog1-K52R and hog1-T174A proteins are tyrosine phosphorylated during osmotic stress (Schüller et al. 1994), they presumably form a complex with Pbs2p, Sholp, and Stellp. If they do form a complex, then scaffolding per se appears not be sufficient to maintain signal specificity. Instead, we propose that phosphorylation of a target protein by Hoglp is responsible for limiting cross talk in wild-type strains.

In principle, the target of Hoglp responsible for limiting cross talk might be any component of the osmolarity-induced cross talk pathway (from Sholp to Ste12p). If Hoglp inhibited a component of the pheromone response MAPK cascade or other proteins necessary for mating, we might expect $1 \mathrm{~m}$ sorbitol to inhibit mating or induction of FU S1::lacZ by $\alpha$-factor. Such inhibition has not been observed (Fig. 2; Table 1; S. O'Rourke, unpubl.). Based on these observations, we suggest that Hoglp may down-regulate components of the HOG pathway, specifically the Sholp branch, after stimulation by high osmolarity. Although we cannot rigorously exclude Ste50p, Ste20p, and Ste11p as targets, a particularly appealing possibility is that Sholp is phosphorylated by Hoglp.

Feedback regulation of receptor activity by protein phosphorylation has been well documented for G-protein-coupled receptors (for review, see Ferguson et al. 1996). We propose that inhibition of the Sholp branch of the HOG pathway ensures that activation of the pathway is transient. Sustained activation of the HOG pathway results in lethal ity (M aeda et al. 1994; Wurgler-M urphy et al. 1997). Thus, mechanisms that down-regulate the pathway after the appropriate responses have occurred are essential. The protein phosphatases Ptp2p and Ptp3p inhibit Hoglp activity (Jacoby et al . 1997; WurglerMurphy et al. 1997), which is sufficient for inhibiting the Ssk1p-dependent branch, as Hoglp is the only known target of this branch. However, if Hoglp were the only point of down-regulation, high osmolarity would cause chronic stimulation of the Sholp branch (including Ste20p, Ste50p, and Ste11p), which could lead to inappropriate activation of the pheromone response and pseudohyphal growth pathways in wild-type cells. We thus propose that Hoglp plays a critical role in adaptation of yeast to high osmolarity conditions by inhibiting the Sholp branch of the HOG pathway.

Hall et al . (1996) reported recently that high osmolarity $(0.5 \mathrm{M} \mathrm{NaCl})$ can induce the pheromone response pathway. These workers observed a twofold increase in phosphorylation of Fus3p on tyrosine-182, which is phosphorylated by Ste7p (Gartner et al. 1992). Strikingly, phosphorylation was increased 13-fold after osmotic shock of a hogl $\Delta$ strain. Hall et al. (1996) interpreted these observations to indicate that high osmolarity induced cross talk and that this cross talk was inhibited by the HOG pathway. Other analyses led them to suggest that this inhibition might be exerted both on Fus3p and on an additional component. We suggest that this inhi- 
bition is mediated by Hoglp acting early in the pathway, perhaps on Sholp itself. Although there are aspects of the observations by Hall et al. (1996) that we do not understand (in particular, why they did not observe induction of FUS1::IacZ by high osmolarity in their hogl strain), we believe that their findings and ours on cross talk in hogl strains concern the same phenomenon. In particular, we propose that the transient activation of Fus3p observed by $\mathrm{Hall}$ et al. (1996) with $0.5 \mathrm{~m} \mathrm{NaCl}$ treatment of wild-type strains represents a normal level of cross activation of the pheromone response pathway that is subsequently damped by Hoglp.

The observation that mutations in HOG1 and PBS2 allow high levels of cross talk explains prior observations, particularly on enhanced pseudohyphal development exhibited by hogl mutants (Madhani et al. 1997) and on increased basal expression of the pheromone response pathway seen in mutants defective in PBS2 (Stevenson et al. 1995). Our discovery that osmolarityinduced cross talk works via the Sholp branch of the HOG pathway made it possible to uncover the role of Ste20p and Ste50p in this branch of the HOG pathway. We anticipate that Sholp may communicate similarly with Ste20p and Ste50p to activate the pseudohyphal development pathway in wild-type cells.

\section{Materials and methods}

Strains, media, and genetic techniques

Yeast strains were grown in YEPD medium (1\% yeast extract, $2 \%$ Bacto-Peptone, $2 \%$ glucose) at $30^{\circ} \mathrm{C}$. Synthetic complete medium (Rose et al. 1990) was used for maintaining plasmids and selecting gene replacements. $\mathrm{D}$-sorbitol and $\mathrm{NaCl}$ (Sigma) were used at final concentrations of 1 or $1.2 \mathrm{M}$ as indicated. For $\alpha$-factor treatments, cells were grown in liquid YEPD medium, and $0.5 \mathrm{mg} / \mathrm{ml} \alpha$-factor in $0.01 \mathrm{M} \mathrm{HCl}$ was added to a final concentration of $0.005 \mathrm{mg} / \mathrm{ml}$. Yeast transformations were done by the lithium acetate procedure (Schiestl and Gietz 1989). Y east strains (T able 3) were derived from the EG 123 strain background (trp1-1 leu2-3, 112 ura3-52 his4 can1; Siliciano and Tatchell 1984), except for the $\Sigma 1278 b$-derived strains SO392, SO393, SO394, and SO395, and the MAT $\alpha$ mating tester (IH1793). The four $\Sigma 1278 \mathrm{~b}$ strains were derivatives of RRY 1045 (MATa/MAT $\alpha$ ura3-52/ura3-52 his3::hisG/his3::hisG leu2:: hisG / LEU 2trpl::hisG/TRP1), which was obtained from G. Fink (Whitehead Institute, MIT, Cambridge, MA). Gene disruptions were confirmed by phenotypic analysis and/or PCR reactions with gene-specific primers.

Escherichia coli strain DH $5 \alpha$ was used for propagation of plasmids (Table 4). To construct the sho1::TRP1 disruption plasmid, a 1.7-kb fragment encompassing the SHO 1 locus from -392 bp from the initiating ATG to +229 bp from the stop codon was amplified from genomic DN A prepared from yeast strain $\mathrm{SO} 329$ by PCR using Expand polymerase in Expand buffer no. 1. The $5^{\prime}$ primer had the sequence ATAGACCCTTGAACCTACATATCCG, and the $3^{\prime}$ primer had the sequence GTCAAGTCAATGACATGAGAGTGC. The PCR product was ligated into the pCR2.1 vector (Invitrogen) to yield pSO19. pSO19 was cleaved with $\mathrm{SnaBI}$ and Clal to remove the entire $\mathrm{SHO} 1$ coding region except for the DNA encoding the 11 carboxy-terminal amino acids. After treating the cleaved pSO19 with the Klenow fragment, a Klenow-treated, 0.85-kb, BamHI TRP1 DN A fragment from YDp-W (Berben et al. 1991) was ligated into the cleaved pSO19 to yield pSO72. The orientation of the TRP1 gene in pSO 72 was determined to be opposite that of the SHO 1 gene. Treatment of pSO72 with Notl and Spel liberated the shol::TRP1 deletion allele that was used for yeast transformations.

The FUS1::IacZ::LEU 2 integrating reporter gene, from plasmid pFC23, was introduced into a wild-type strain (IH1783), which was crossed to other strains to create isogenic strains with respect to the FU S1::IacZ::LEU 2 gene. For creating the FU S1::lacZ::Ieu2s reporter, plasmid p307 (LEU 2::U RA 3::leu2 $\Delta$ ) was transformed into yeast containing the FU S1::IacZ::LEU 2 reporter and plated on synthetic complete medium lacking uracil. Strains were then patched onto 5-fluoro-orotic acid plates to select for loopouts of the U RA 3 gene (Boeke et al. 1984). A leu2 isolate was used in subsequent crosses to obtain strains containing the FU S1::IacZ::Ieu2 $\Delta$ reporter gene. The activity of the FU S1::lacZ reporter gene was identical when marked with either the LEU 2 or leu2 $\Delta$ allele.

\section{$\beta$-Galactosidase assays}

LacZ expression was measured as described previously (Stern et al. 1984), except that log-phase cells were treated for 5 or $6 \mathrm{hr}$ as indicated by diluting into fresh medium, medium containing $\alpha$-factor, or medium containing $1 \mathrm{~m}$ sorbitol prior to harvesting.

Yeast mutagenesis and screening for hogl-like mutants

A FU S1::IacZ a strain (SO329) was mutagenized with methanesulfonic acid ethyl ester (Sigma) in four separate pools to $22 \%$ $86 \%$ killing and plated on YEPD plates at a density of $\sim 300$ colonies per plate (Lawrence 1991). After growth for 3-7 days, colonies were patched onto a YEPD plate at a density of 50/ plate. The plates were incubated at $30^{\circ} \mathrm{C}$ overnight and replica plated to a N o. 3 Whatman filter on a YEPD $+1 \mathrm{~m}$ sorbitol plate and grown overnight. $\beta-G$ alactosi dase assays were performed on the filters to identify strains expressing FU S1::IacZ. Of 2400 strains examined, 16 were isolated that induced FU S1::IacZ in response to $1 \mathrm{~m}$ sorbitol. These were tested for complementation of the FUS1::lacZ induction phenotype by HOG1- and PBS2-containing plasmids (Brewster et al . 1993). The sensitivity of the screen was demonstrated by the isolation of one apparent hogl mutant that exhibited $22 \%$ of the cross talk observed for the hogl deletion strain.

\section{Quantitative mating analysis}

$M$ ating efficiencies were performed as described (Valtz and Herskowitz 1996), except that $6.0 \times 10^{6}$ log-phase cells were used per mating reaction, and mating reactions were performed for 24 $\mathrm{hr}$. Additionally, sterile filters containing the mating mixes were cut in half: One-half was incubated on YEPD, and the other half was incubated on YEPD $+1 \mathrm{M}$ sorbitol.

\section{Microscopy}

Yeast cells were photographed with a Zeiss axioskop microscope with a $100 \times$ objective lens. Colonies on plates were photographed with a Zeiss Axioskop microscope using a 10× objective lens.

\section{Detection of phosphorylated Hoglp}

To assay Hoglp activation, $1.0 \mathrm{OD}_{600}$ unit of log phase yeast was harvested for each strain and concentrated in $1 \mathrm{ml}$ of YEPD medium. For $\mathrm{N} \mathrm{aCl}$ treatments, $0.25 \mathrm{ml}$ of $3.5 \mathrm{M} \mathrm{N} \mathrm{aCl}$ was added to the cells for $5 \mathrm{~min}$ at room temperature in microcentrifuge tubes. Protein extracts were prepared by centrifuging the tubes 
O'Rourke and Herskowitz

Table 3. Strains used

\begin{tabular}{|c|c|c|}
\hline Strain & Relevant genotype & Source \\
\hline $\mathrm{IH} 1793$ & MAT $\alpha$ Iys1 & $\begin{array}{l}\text { Ira Herskowitz }(\mathrm{IH}) \\
\text { collection }\end{array}$ \\
\hline $\mathrm{IH} 2731$ & MATa ste5::LEU 2 & IH collection \\
\hline $\mathrm{IH} 2735$ & MATa ste20::TRP1 & IH collection \\
\hline SO 143 & MATa hogl::hisG ste12::TRP1 FU S1::IacZ::LEU 2 & this study \\
\hline SO329 & MATa FU S1::IacZ::LEU 2 & this study \\
\hline SO330 & MATa hog1::hisG FU S1::lacZ::leu2 & this study \\
\hline SO331 & MATa hog1::hisG ste4::LEU 2 FU S1::IacZ::Ieu2 & this study \\
\hline SO332 & MATa hog1::hisG ste5::LEU 2 FU S1::lacZ::LEU 2 & this study \\
\hline SO333 & MATa hog1::hisG stell::U RA3 FU S1::IacZ::LEU 2 & this study \\
\hline SO334 & MATa hog1::hisG ste7::LEU 2 FU S1::lacZ::Ieu2 & this study \\
\hline SO335 & MATa hog1::hisG fus3::U RA3 kss1::U RA3 FU S1::lacZ::leu2 & this study \\
\hline SO336 & MATa ste11::U RA3 FU S1::lacZ::leu2 & this study \\
\hline SO341 & MATa ste4::LEU 2 FU S1::lacZ::leu2 & this study \\
\hline SO344 & MATa hog1::hisG sho1::TRP1 ste4::LEU 2 FU S1::IacZ::leu2 & this study \\
\hline SO351 & MATa sho1::TRP1 FU S1::lacZ::leu2 & this study \\
\hline SO352 & MATa ssk 1::LEU 2 FU S1::lacZ::leu2 & this study \\
\hline SO353 & MATa ssk1::LEU 2 sho1::TRP1 FU S1::IacZ::leu2 & this study \\
\hline SO354 & MATa ssk2::LEU 2 ssk22::LEU 2 FU S1::lacZ::leu2 & this study \\
\hline SO355 & MATa ssk2::LEU 2 sk22::LEU 2 sho1::TRP1 FU S1::IacZ::Ieu2 & this study \\
\hline SO356 & MATa hog1::hisG::U RA 3::hisG sho1::TRP1 FU S1::lacZ::leu2 & this study \\
\hline SO357 & MATa hog1::hisG::URA3::hisG ssk1::LEU 2 sho1::TRP1 FU S1::lacZ::leu2 & this study \\
\hline SO358 & MATa hogl::hisG ::U RA 3::hisG ssk2::LEU 2 ssk22::LEU 2 FU S1::IacZ::leu2 & this study \\
\hline SO359 & MATa hog1::hisG::U RA 3::hisG ssk2::LEU 2 ssk22::LEU 2 sho1::TRP1 FU S1::lacZ::leu2 & this study \\
\hline SO360 & MATaste50::hisG::U RA3::hisG FU S1::lacZ::leu2 & this study \\
\hline SO361 & MATa ste50::hisG::U RA 3::hisG ssk1::LEU 2 FU S1::lacZ::leu2 & this study \\
\hline SO373 & MATa hog1::hisG ste50::hisG::URA3::hisG FU S1::lacZ::leu2 & this study \\
\hline SO382 & MATa pbs2::LEU 2 FU S1::IacZ::Ieu2 & this study \\
\hline SO383 & MATa hogl::hisG::U RA 3::hisG pbs2::LEU 2 FU S1::lacZ::leu2 & this study \\
\hline SO384 & MATa hog1::hisG::U RA 3::hisG ssk1::LEU 2 FU S1::lacZ::leu2 & this study \\
\hline SO385 & MATa gpd1::TRP1 FU S1::IacZ::LEU 2 & this study \\
\hline SO387 & MATa ssk1::LEU 2 ste20::TRP1 FU S1::IacZ::LEU 2 & this study \\
\hline SO391 & MATa hog1::hisG ste20::TRP1 FU S1::lacZ::LEU 2 & this study \\
\hline SO392 & MATa/MAT $\alpha$ leu2/LEU 2 & this study \\
\hline SO393 & MATa/MAT $\alpha$ leu2/LEU 2 hogl::hisG::U RA3::hisG/hogl::hisG ::U RA3::hisG & this study \\
\hline SO394 & MATa/MAT $\alpha$ leu2/LEU 2 sho1::TRP1/sho1::TRP1 & this study \\
\hline SO395 & MATa/MAT $\alpha$ leu2/LEU 2 sho1::TRP1/sho1::TRP1 hog1::hisG ::U RA3::hisG/hog1::hisG::U RA 3::hisG & this study \\
\hline SO398 & MATa hog1::hisG::U RA 3::hisG ste5::LEU 2 sho1::TRP1 & this study \\
\hline SO399 & MATa ste11::ura3::TRP1 ssk1::LEU 2 FU S1::lacZ::leu2 & this study \\
\hline
\end{tabular}

Strains SO 392-SO 395 are in the $\Sigma 1278$ b strain background, whose full genotype is trpl leu2 ura3 his3. All other strains (except IH 1793) are in the EG123 strain background, whose full genotype is trp1 leu2 ura3 his4 can1.

for $30 \mathrm{sec}$ and aspirating the supernatants. Cell pellets were incubated on ice for $5 \mathrm{~min}$ prior to resuspension in $150 \mu \mathrm{l}$ of 1.85 $\mathrm{M} \mathrm{N} \mathrm{aOH}, 7.4 \% \beta$-mercaptoethanol. After incubation for $10 \mathrm{~min}$ on ice, $150 \mu \mathrm{l}$ ice-cold 50\% TCA was added. Samples were incubated on ice for $10 \mathrm{~min}$ followed by centrifugation at $4^{\circ} \mathrm{C}$ for $2 \mathrm{~min}$. Pellets were washed with $1 \mathrm{ml}$ of ice-cold acetone, centrifuged at $4^{\circ} \mathrm{C}$ for $2 \mathrm{~min}$, and resuspended in $100 \mu \mathrm{l}$ of $1 \times$ sample buffer containing $1 \mathrm{mM} \mathrm{N} \mathrm{a}_{3} \mathrm{VO}_{4}$. After boiling the samples for 5 min and briefly centrifuging, $15 \mu \mathrm{l}$ was run on a $10 \%$ polyacrylamide gel that was transferred to a nitrocellulose membrane. After blocking the nitrocellulose in 5\% BSA, $20 \mathrm{~mm}$ Tris (pH 7.5), $428 \mathrm{~mm} \mathrm{NaCl}, 0.2 \%$ Tween 20 (TBST) for $1 \mathrm{hr}$ at room temperature, the membrane was probed with the anti-phosphospecific p38 antibody (N ew England Biolabs) in TBST overnight. Immunoreactivity was localized with the ECL system (Amersham) using horseradish peroxidase-conjugated anti-rabbit secondary antibodies.

\section{Acknowledgments}

We thank Michael Gustin, Linda Huang, Massoud Ramezani Rad, and Haruo Saito for generously providing plasmids, Bruce Cree, Aaron Neiman, and Cora Styles for strains, and Gerry Fink for personal communication. We also thank members of our laboratory, Shaun Coughlin and Wendell Lim, for valuable discussion, and Flora Banuett, Linda Huang, Kenji Irie, Doug Jeffery, and Mary Maxon, for comments on the manuscript. This work was supported by $\mathrm{N}$ ational Institutes of Health (NIH) grant AI18738 (to I. H.). S.O'R. was supported by an NIH training grant, the Markey Program in Biological Sciences, and the Herbert W. Boyer Fund.

The publication costs of this article were defrayed in part by payment of page charges. This article must therefore be hereby marked "advertisement" in accordance with 18 USC section 1734 solely to indicate this fact. 
Table 4. Plasmids used

\begin{tabular}{|c|c|c|}
\hline Plasmid & Description & Source/Reference \\
\hline pRS316 & CEN6 URA3 & Sikorski and Hieter (1989) \\
\hline pJB15 & pRS316 + HOG1 & Brewster et al. (1993) \\
\hline pJBM 3 & pRS316 + hogl-K52R & Schüller et al. (1994) \\
\hline pJBM 4 & pRS316 + hogl-T174A & Schüller et al. (1994) \\
\hline pJBM 2 & pRS316 + hogl-Y176F & Schüller et al. (1994) \\
\hline pJB41 & pRS316 + PBS2 & Brewster et al. (1993) \\
\hline pste4::LEU 2 & ste4::LEU 2 & Whiteway et al. (1989) \\
\hline pDM4U & ste50::hisG::U RA3::hisG & Xu et al. (1996) \\
\hline pNC202 & stell::U RA3 & Beverly Errede (University of N orth Carolina, Chapel Hill) \\
\hline $\mathrm{pDH} 90$ & ste7::LEU 2 & D. Higgins and K. Tatchell (Louisiana State University, Baton Rouge) \\
\hline pBC99 & hogl::hisG::U RA3::hisG & Bruce Cree/Ira Herskowitz $(\mathrm{IH})$ collection \\
\hline pMA11 & pbs2::LEU 2 & Brewster et al. (1993) \\
\hline pSO 72 & shol::TRP1 & this study \\
\hline pDSS14 & ssk1::LEU 2 & H. Saito (Dana-Farber Cancer Institute, Boston, M A) \\
\hline pDSS24 & ssk2::LEU 2 & H. Saito \\
\hline pDYC734 & ssk22::LEU 2 & H. Saito \\
\hline pU Cgpd1::TRP1 & gpd1::TRP1 & Albertyn et al. (1994) \\
\hline pFC23 & FU S1::IacZ::LEU 2 & Fred Chang/IH collection \\
\hline p307 & leu $2 \Delta:: U$ RA3 & Phil Hieter (University of British Columbia, Vancouver, Canada) \\
\hline pUT 11 & ura3s::TRP1 & Cross (1997) \\
\hline YCplac22 & CEN4 TRP1 & Gietz and Sugino (1988) \\
\hline
\end{tabular}

\section{References}

Albertyn, J., S. Hohmann, J.M. Thevelein, and B.A. Prior. 1994. GPD 1, which encodes glycerol-3-phosphate dehydrogenase, is essential for growth under osmotic stress in Saccharomyces cerevisiae, and its expression is regulated by the highosmolarity glycerol response pathway. Mol. Cell. Biol. 14: 4135-4144.

Banuett, F. 1998. Signalling in the yeasts: An informational cascade with links to the filamentous fungi. Microbiol. Mol. Biol. Rev. 62: 249-274.

Bardwell, L., J.G. Cook, C.J. Inouye, and J. Thorner. 1994. Signal propagation and regulation in the mating pheromone re sponse pathway of the yeast Saccharomyces cerevisiae. Dev. Biol. 166: 363-379.

Berben, G., J. Dumont, V. Gilliquet, P.A. Bolle, and F. Hilger. 1991. The YDp plasmids: A uniform set of vectors bearing versatile gene disruption cassettes for Saccharomyces cerevisiae. Yeast 7: 475-477.

Blomberg, A. and L. Adler. 1992. Physiology of osmotolerancein fungi. Adv. Microbiol. Physiol. 33: 145-212.

Boeke, J.D., F. LaCroute, and G.R. Fink. 1984. A positive selection for mutants lacking orotidine-5'-phosphate decarboxylase activity in yeast: 5 -fluoro-orotic acid resistance. Mol. \& Gen. Genet. 197: 345-346.

Brewster, J.L., T. de Valoir, N.D. Dwyer, E. Winter, and M.C. Gustin. 1993. An osmosensing signal transduction pathway in yeast. Science 259: 1760-1763.

Cook, J.G., L. Bardwell, S.J. Kron, and J. Thorner. 1996. Two novel targets of the M AP kinase Kssl are negative regulators of invasive growth in the yeast Saccharomyces cerevisiae. Genes \& Dev. 10: 2831-2848.

Cook, J.G., L. Bardwell, and J. Thorner. 1997. Inhibitory and activating functions for MAPK Kss1 in the S. cerevisiae filamentous-growth signalling pathway. Nature 390: 85-88.

Cross, F.R. 1997. “M arker swap" plasmids: Convenient tools for budding yeast molecular genetics. Yeast 13: 647-653.

Davenport, K.R., M. Sohaskey, Y. Kamada, D.E. Levin, and M .C. Gustin. 1995. A second osmosensing signal transduction pathway in yeast. Hypotonic shock activates the PKC1 protein kinase-regulated cell integrity pathway. J. Biol. Chem. 270: 30157-30161.

Dietzel, C. and J. Kurjan. 1987. The yeast SCG1 gene: $\alpha \mathrm{G} \alpha$-like protein implicated in the a and $\alpha$-factor response pathway. Cell 50: 1001-1010.

Errede, B., A. Gartner, Z. Zhou, K. N asmyth, and G. Ammerer. 1993. MAP kinase-related FUS3 from S. cerevisiae is activated by STE7 in vitro. Nature 362: 261-264.

Feng, Y., L.Y. Song, E. Kincaid, S.K. Mahanty, and E.A. Elion. 1998. Functional binding between $\mathrm{G} \beta$ and the LIM domain of Ste5 is required to activate the MEKK Stell. Curr. Biol. 8: 267-278.

Ferguson, S.S., L.S. Barak, J. Zhang, and M.G. Caron. 1996. Gprotein-coupled receptor regulation: Role of G-proteincoupled receptor kinases and arrestins. Can. J. Physiol. Pharmacol. 74: 1095-1110.

Gartner, A., K. N asmyth, and G. Ammerer. 1992. Signal transduction in Saccharomyces cerevisiae requires tyrosine and threonine phosphorylation of FUS3 and KSS1. Genes \& Dev. 6: $1280-1292$.

Gietz, R.D. and A. Sugino. 1988. New yeast-Escherichia coli shuttle vectors constructed with in vitro mutagenized yeast genes lacking six-base pair restriction sites. Gene 74: 527534.

Gimeno, C.J., P.O. Ljungdahl, C.A. Styles, and G.R. Fink. 1992. Unipolar cell divisions in the yeast S. cerevisiae lead to filamentous growth: Regulation by starvation and RAS. Cell 68: 1077-1090.

Hall, J.P., V. Cherkasova, E. Elion, M.C. Gustin, and E. Winter. 1996. The osmoregulatory pathway represses mating pathway activity in Saccharomyces cerevisiae: Isolation of a FU S3 mutant that is insensitive to the repression mechanism. Mol. Cell. Biol. 16: 6715-6723.

Herskowitz, I. 1995. M AP kinase pathways in yeast: for mating and more. Cell 80: 187-197.

Inouye, C., N. Dhillon, and J. Thorner. 1997. Ste5 RING-H2 domain: Role in Ste4-promoted oligomerization for yeast pheromone signaling. Science 278: 103-106. 
Jacoby, T., H. Flanagan, A. Faykin, A.G. Seto, C. Mattison, and I. Ota. 1997. Two protein-tyrosine phosphatases inactivate the osmotic stress response pathway in yeast by targeting the mitogen-activated protein kinase, Hogl. J. Biol. Chem. 272: 17749-17755.

Kamada, Y., U.S. Jung, J. Piotrowski, and D.E. Levin. 1995. The protein kinase C-activated MAP kinase pathway of Saccharomyces cerevisiae mediates a novel aspect of the heat shock response. Genes \& Dev. 9: 1559-1571.

Larsson, K., R. Ansell, P. Eriksson, and L. Adler. 1993. A gene encoding sn-glycerol 3-phosphate dehydrogenase (NAD ${ }^{+}$) complements an osmosensitive mutant of Saccharomyces cerevisiae. Mol. Microbiol. 10: 1101-1111.

Lawrence, C.W. 1991. Classical mutagenesis techniques. In Guide to yeast genetics and molecular biology (ed. C. Guthrie and G.R. Fink), pp. 273-281. Academic Press, San Diego, CA.

Leberer, E., D.Y. Thomas, and M. Whiteway. 1997. Pheromone signalling and polarized morphogenesis in yeast. Curr. O pin. Genet. Dev. 7: 59-66.

Leeuw, T., C. Wu, J.D. Schrag, M. Whiteway, D.Y. Thomas, and E. Leberer. 1998. Interaction of a G-protein $\beta$-subunit with a conserved sequence in Ste20/PAK family protein kinases. Nature 391: 191-195.

Levin, D.E. and B. Errede. 1995. The proliferation of M AP kinase signaling pathways in yeast. Curr. Opin. Cell Biol. 7: 197202.

Liu, H., C.A. Styles, and G.R. Fink. 1993. Elements of the yeast pheromone response pathway required for filamentous growth of diploids. Science 262: 1741-1744.

Madhani, H.D., C.A. Styles, and G.R. Fink. 1997. M AP kinases with distinct inhibitory functions impart signaling specificity during yeast differentiation. Cell 91: 673-684.

Maeda, T., S.M. Wurgler-M urphy, and H. Saito. 1994. A twocomponent system that regulates an osmosensing MAP kinase cascade in yeast. $N$ ature 369: 242-245.

Maeda, T., M. Takekawa, and H. Saito. 1995. Activation of yeast PBS2 MAPKK by MAPKKKs or by binding of an SH3-containing osmosensor. Science 269: 554-558.

McCaffrey, G., F.J. Clay, K. Kelsay, and G.F. Sprague, Jr. 1987. Identification and regulation of a gene required for cell fusion during mating of the yeast Saccharomyces cerevisiae. Mol. Cell. Biol. 7: 2680-2690.

Miyajima, I., M. N akafuku, N. Nakayama, C. Brenner, A. M iyajima, K. Kaibuchi, K. Arai, Y. Kaziro, and K. Matsumoto. 1987. GPA1, a haploid-specific essential gene, encodes a yeast homolog of mammalian $G$ protein which may be involved in mating factor signal transduction. Cell 50: 10111019.

Neiman, A.M. and I. Herskowitz. 1994. Reconstitution of a yeast protein kinase cascade in vitro: Activation of the yeast MEK homologue STE7 by STE11. Proc. Natl. Acad. Sci. 91: 3398-3402.

Posas, F. and H. Saito. 1997. Osmotic activation of the HOG MAPK pathway via Ste1lp MAPKKK: Scaffold role of Pbs2p MAPKK. Science 276: 1702-1705.

- - - 1998. Activation of the yeast SSK2 MAP kinase kinase kinase by the SSK 1 two-component response regulator. EMBO J. 17: 1385-1394.

Ramer, S.W. and R.W. Davis. 1993. A dominant truncation allele identifies a gene, STE20, that encodes a putative protein kinase necessary for mating in Saccharomyces cerevisiae. Proc. Natl. Acad. Sci. 90: 452-456.

Roberts, R.L. and G.R. Fink. 1994. Elements of a single MAP kinase cascade in Saccharomyces cerevisiae mediate two de velopmental programs in the same cell type: Mating and invasive growth. Genes \& Dev. 8: 2974-2985.

Robinson, M.J. and M.H. Cobb. 1997. Mitogen-activated protein kinase pathways. Curr. Opin. Cell Biol. 9: 180-186.

Rose, M.D., F. Winston, and P. Hieter. 1990. Methods in yeast genetics: A laboratory course manual. Cold Spring Harbor Laboratory Press, Cold Spring Harbor, N.Y.

Schiestl, R.H. and R.D. Gietz. 1989. High efficiency transformation of intact yeast cells using single stranded nucleic acids as a carrier. Curr. Genet. 16: 339-346.

Schrick, K., B. Garvik, and L.H. Hartwell. 1997. Mating in Saccharomyces cerevisiae: The role of the pheromone signal transduction pathway in the chemotropic response to pheromone. Genetics 147: 19-32.

Schüller, C., J.L. Brewster, M.R. A lexander, M.C. Gustin, and H. Ruis. 1994. The HOG pathway controls osmotic regulation of transcription via the stress response el ement (STRE) of the Saccharomyces cerevisiae CTT1 gene. EMBO J. 13: 43824389.

Sikorski, R.S. and P. Hieter. 1989. A system of shuttle vectors and yeast host strains designed for efficient manipulation of DN A in Saccharomyces cerevisiae. Genetics 122: 19-27.

Siliciano, P.G. and K. Tatchell. 1984. Transcription and regulatory signals at the mating type locus in yeast. Cell 37: 969978.

Stern, M., R. Jensen, and I. Herskowitz. 1984. Five SWI genes are required for expression of the $\mathrm{HO}$ gene in yeast. J. Mol. Biol. 178: 853-868.

Stevenson, B.J., B. Ferguson, C. DeVirgilio, E. Bi, J.R. Pringle, G. Ammerer, and G.F. Sprague, Jr. 1995. Mutation of RGA1, which encodes a putative GTPase-activating protein for the polarity-establishment protein Cdc42p, activates the pheromone-response pathway in the yeast Saccharomyces cerevisiae. Genes \& Dev. 9: 2949-2963.

Tedford, K., S. Kim, D. Sa, K. Stevens, and M. Tyers. 1997. Regulation of the mating pheromone and invasive growth responses in yeast by two M AP kinase substrates. Curr. Biol. 7: 228-238.

Valtz, N. and I. Herskowitz. 1996. Pea2 protein of yeast is localized to sites of polarized growth and is required for efficient mating and bipolar budding. J. Cell Biol. 135: 725-739.

Whiteway, M., L. Hougan, D. Dignard, D.Y. Thomas, L. Bell, G.C. Saari, F.J. Grant, P. O'Hara, and V.L. MacKay. 1989. The STE4 and STE18 genes of yeast encode potential $\beta$ and $\gamma$ subunits of the mating factor receptor-coupled $G$ protein. Cell 56: 467-477.

Whiteway, M.S., C. Wu, T. Leeuw, K. Clark, A. Fourest-Lieuvin, D.Y. Thomas, and E. Leberer. 1995. Association of the yeast pheromone response $G$ protein $\beta \gamma$ subunits with the MAP kinase scaffold Ste5p. Science 269: 1572-1575.

Wu, C., M. Whiteway, D.Y. Thomas, and E. Leberer. 1995. M olecular characterization of Ste20p, a potential mitogen-activated protein or extracellular signal-regulated kinase kinase (MEK) kinase kinase from Saccharomyces cerevisiae. J. Biol. Chem. 270: 15984-15992.

Wurgler-M urphy, S.M., T. M aeda, E.A. Witten, and H. Saito. 1997. Regulation of the Saccharomyces cerevisiae HOG1 mitogen-activated protein kinase by the PTP2 and PTP3 protein tyrosine phosphatases. Mol. Cell. Biol. 17: 1289-1297.

Xu, G., G. Jansen, D.Y. Thomas, C.P. Hollenberg, and M. Ramezani Rad. 1996. Ste50p sustains mating pheromone-induced signal transduction in the yeast Saccharomyces cerevisiae. Mol. Microbiol. 20: 773-783.

Yashar, B., K. Irie, J.A. Printen, B.J. Stevenson, G.F. Sprague, Jr., K. Matsumoto, and B. Errede. 1995. Y east MEK-dependent signal transduction: Response thresholds and parameters affecting fidelity. Mol. Cell. Biol. 15: 6545-6553. 


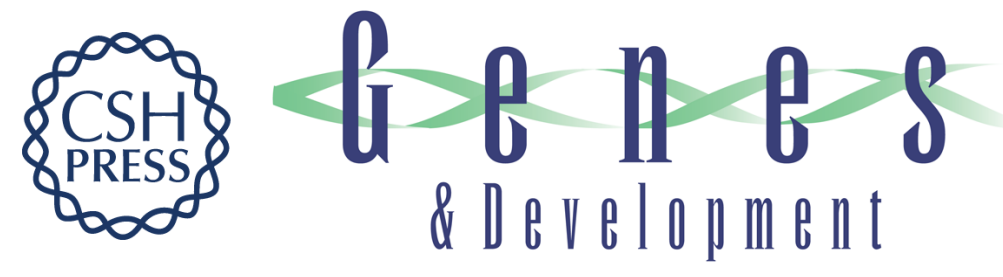

\section{The Hog1 MAPK prevents cross talk between the HOG and pheromone response MAPK pathways in Saccharomyces cerevisiae}

Sean M. O'Rourke and Ira Herskowitz

Genes Dev. 1998, 12:

Access the most recent version at doi:10.1101/gad.12.18.2874

References This article cites 54 articles, 26 of which can be accessed free at: http://genesdev.cshlp.org/content/12/18/2874.full.html\#ref-list-1

License

Email Alerting

Receive free email alerts when new articles cite this article - sign up in the box at the top Service right corner of the article or click here.

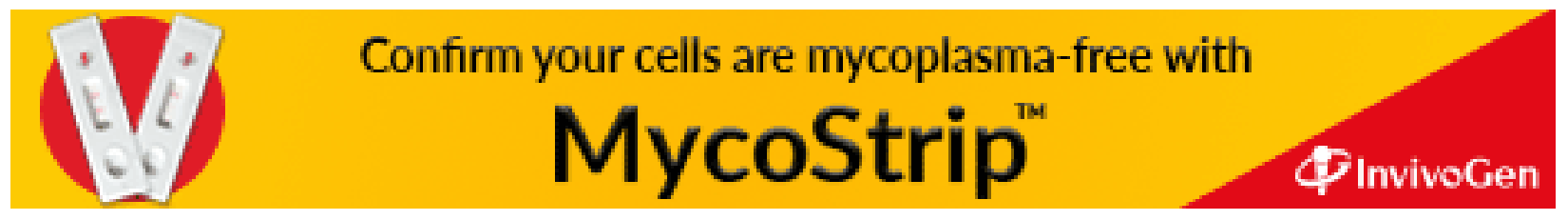

